\title{
INDICATIONS AND CONTRA-INDICATIONS FOR TREATING TEETH INVOLVED WITH PYORRHEA ALVEOLARIS.
}

\author{
Including Statements Having Reference to the Various Suggested Remedies, Including \\ Emetin.-Discussion Not to Include the Technic of Instrumentation or the Care \\ of the Tooth Surfaces by the Dentist or Patient.
}

By J. D. Patterson, D. D. S., Kansas City, Mo.

(Read before the National Dental Association at its Twentieth Annual Session, Louisville, Ky., July 25-28, 1916.)

$\mathrm{I}^{\mathrm{N}}$ N THE treatment of Pyorrhea, or in the treatment of any condition of disease where infectious products are in evidence, attention should be given to the possibility of the spread of such infection, and then to determine whether or no such poisoning can be positively prevented.

The first consideration at all times should be the patients' well being, which demands that he be freed from the dangers of chronic infection or suppuration. The members of the dental profession have in the past, and are yet, too prone to look leniently upon evidence of chronic inflammation which they have been unable to correct.

These chronic infectious suppurative conditions, it has been reasonably proved in many cases, have as a corollary, diseased conditions elsewhere in the body, and this holds good even when patients are unaware that obscure infection abounds.

However, it must also be said that the wholesale removal of suspected teeth must ever wait upon the operators' judgment and that judgment must depend on diagnostic ability of the highest type as a prerequisite before extraction or before treatment.

Indications and contra-indications for treatment in Pyorrhea as well as in all other pathological conditions of the oral cavity, must therefore be based upon good theory, correct judgment, and wide experience.

The question is-I apprehend-shall teeth be removed? or shall they be treated, so as to give in utility, comfort and beauty, enough use to justify efforts for their retention?

Upon a broad basis of correct practice, it may be said of Pyorrhea just as of other dental diseases-viz: "teeth which cannot be freed from discomfort and infection should be extracted."

To be able to diagnose and prognose regarding a tooth or teeth affected with pyorrhea demands quite a fund of ability and experience on the part of the operator, and even then, events often prove that errors have crept into our decisions.

The operator-after long clinical experience and close observation-can more clearly come to a correct decision and his opinion is worth more than he who argues from a theoretical standpoint solely, and yet the experienced clinician often is floored because of the Protein causes, symptoms, and environments of this disease. There are so many factors leading to success, and so many factors leading to failure in Pyor- 
rhea treatment, that close study of each case is demanded of the operator.

The "contra-indications" in treatment are many.

1st. If a tooth has no remaining normal attachment it should be removed.

2nd. If the pocket or pockets extend to the apex of a tooth or teeth the pulp. usually is involved-and the root is indented with absorption. This condition contra-indicates treatment and extraction is advised. Operators often excise the root end when this condition exists, but the writer's experience in this operation does not encourage. The per cent of failures to bring about perfect health about an excised root is too great.

To ascertain whether Osteo-clasis has burrowed the root, can usually be determined by delicate but rigid probes, or by the X-ray.

3rd. Antrum involvent. Not infrequently Pyorrhea pockets extending to the apex of bicuspids and molars in the Superior Maxilla result in death of the pulp and subsequently is found abscess of the maxillary sinus.

This condition in Pyorrhea demands the removal of the tooth for there has been too long continued disease to hope for successful treatment.

There are other "contra-indications" but which do not necessarily demand the sacrifice of involved teeth such as:

1st. Teeth having no antagonists. Such teeth on account of non-use fall a prey to disease rapidly-yet can be successfully treated by supplying antagonists and by unusual hygiene care.

2nd. Nasal Catarrh increases the difficulty of treatment, is often caused by Pyorrhea and no doubt Catarrh at times is the inception of Pyorrhea. Treatment must be with the aid of the Rhinologist.

3rd. Auto intoxication from over-eating greatly increases the difficulty of successful Pyorrhea treatment. Indeed the author has often found the utter hopelessness of treatment in pronounced cases of this kind, for "once a glutton always a glutton" is a pretty fair statement.

Some "contra-indications" are not discovered until some weeks or months after treatment is instituted, viz: lack of cooperation on patient's part. There is no more radical contra-indication than the lack of the patient's cooperation.

Neglect of mouth sanitation which has gone on from childhood to adult lifeand which is the prime cause of Pyorrhea, is very difficult to correct, many times quite impossible, especially with men. Pride sometimes sways the woman into change of habit but if improve. ment is not seen, continuation of the treatment is contra-indicated.

Your committee, in asking me to speak upon this subject, have also said, "to include statements having reference to the various suggested remedies, including emetine."

It may be said that request embraces:

1st. Vaccine treatment, either with Autogenous or Stock vaccines.

2nd. The emetine treatment either locally or hypodermically.

3rd. The Succinimid of Mercury treatment.

4th. The Dunlop treatment. Regarding the Vaccine treatment I think my hearers would justly criticise, if I repeated my opinion given of vaccine treatment for this disease which I gave at the Rochester meeting. These opinions have not been changed or but little modified.

Regarding treatment by Emetine $\mathrm{Hy}$ drochloride. After trial and observation, I do not dignify it, by ascribing to it any virtue whatever.

About the treatment with Succinimid of Mercury, I know nothing, practically.

The Dunlop treatment seems to the writer to be entirely empirical, and hasty empiricism at that. The science or logic of this treatment seems to halt.

The writer for nearly 40 years has ob. served the wonderful results attained by careful and persistent surgical and local treatment, (at times re-enforced 
with specific systemic treatment by the the patient's physician to remove constitutional hindrances resulting from faulty metabolism), and needs no doubtful psychological assistance in the treatment of

\section{PYORRHEA ALVEOLARIS.}

The "key note" of treatment is, and the writer thinks, ever will be, to remove out of nature's way every one of the manifold local causes for this condition. Sir Horace Plunket says:

"The time has come when mischievous subordination of physiology to psychology should be ended." "And that the inculcation of faith in nature's healing processes is a safer and surer road to health than psycho-therapy, or serum therapy, with the vista of illusion, and the opportunity for professional charlatanry which it opens up."

Summing up the "various suggested remedies, including Emetine," which your committee asked me to notice, I think it would be very well to recall that in times past our profession has paid a fearsome price for some "gold brick" which soon had the gilding rubbed off, and to scrutinize carefully supposed sure cures and supposed vital facts which have been showered upon us by apostles of the "Shedding of Knowledge."

The following quotation from "Life" is apropos:

"Sometimes boys throw mud at the side of a barn, and as long as the mud remains plastic it holds to the wood fairly well; but when the sun comes out it dries and crumbles and falls off, and the next shower removes all traces of it.

That side of a bari is a good deal like our minds, and the boy may be likened to the modern maker of knowledge. In these days we are pelted pretty hard by plastic facts, but few of them stick. Rerhaps, after all, this is a healthy dispensation of Providence which enables us to shed knowledge. Suppose we were obliged to retain all the "vital" facts that were thrown at us. What an intolerable burden!"

To this quotation I might add, that the "various suggested remedies, including Emetine" would not only be an "intolerable burden" to the operator but a step to insanity for the patient. 